\title{
The Ferret: An Animal Model to Study Influenza Virus
}

John A. Maher, MS, MBA, CMAR, RLATG, and Joanne DeStefano MS, LVT, LAT

There has been much critical influenza research conducted in a little-known laboratory animal-the ferret. The authors review some of these findings, discuss the reasons the ferret often becomes a model for influenza infection, and compare the ferret with other animal models.
Maher is Senior Manager, BioResources and DeStefano is Senior Research Scientist, Vaccines Discovery, Wyeth Research, Pearl River, NY. Please address correspondence to Maher at MaherJ2@wyeth.com.
Human influenza is a viral respiratory disease that often results in a high morbidity and considerable mortality rate in both humans and domestic animals ${ }^{1,2}$. Influenza epidemics vary in severity from year to year. This disease can also wreak economic havoc on a community because of the number of workdays lost to illness and the resulting impact on business ${ }^{2,3}$. Therefore, studying the influenza virus is of considerable importance, both for the discovery of practical treatments for the disease and for the development of vaccines to prevent $\mathrm{it}^{4}$.

\section{Viral Characteristics}

Influenza viruses are helical, enveloped, single-stranded RNA viruses of the family Orthomyxoviridae. Presently, there are three known types-influenza A, B, and $\mathrm{C}$-letter designations that are based on antigenic differences between the viral nucleoproteins (NPs) and matrix (M) proteins. The viruses have an outer lipoprotein envelope and an internal helical ribonucleoprotein core, termed the nucleocapsid, which contains the viral RNA genome and associated proteins. The influenza A virus, which is the prototype virus of the Orthomyxoviridae, contains ten genes distributed among eight RNA segments. One or two genes reside on each of these separate RNA strands $s^{1,5-7}$.

The envelope has two important surface glycoproteins: hemagglutinin (HA) and neuraminidase (NA). Inside the lipoprotein envelope is the shell, comprising the M protein. The M protein encloses the viral RNA, which is associated with the NP and other proteins that function in replication and transcription ${ }^{1,5-7}$.

The glycoprotein HA is responsible for attachment to the cell before penetration and fusion, and cells infected with influenza often express the proteins HA, NA, NP, and M on their surface. The HA protein has a tendency to undergo antigenic changes, due most commonly to antigenic drift-that is, the accumulation of point mutations over time. Alternately, changes to the genome can also result from antigenic shift-the occasional genetic reassortment between human influenza $A$ and strains from species other than humans ${ }^{1,5,6,8}$. These frequent genomic changes result in phenotypic changes that add to the challenge in developing treatments and vaccines for this disease.

\section{The Ferret Model}

The ferret's usefulness as a model for influenza became apparent in 1933, when influenza-induced rhinitis was first observed in the ferret ${ }^{7,9}$. Since then there has been extensive use of the ferret for studying various aspects of human influenza viral infection and its course of action. The development of many of the contemporary concepts of immunity to the influenza virus would have been impossible without the use of the ferret ${ }^{1,7}$.

The ferret has proven to be a good model for studying influenza for several reasons:

- Influenza infection in the ferret closely resembles that in humans with respect to clinical signs, pathogenesis, and immunity;

- Types A and B of human influenza virus naturally infect the ferret, thus providing an opportunity to study a completely controlled population in which 
to observe the interplay of transmission of infection, illness, and sequence variation of amino acids in the glycoproteins of the influenza virus; and

- The ferret has other physical characteristics that make it an ideal model for deciphering the manifestations of the disease $^{10}$.

\section{Symptoms and Clinical Course}

Ferrets and humans show very similar clinical signs of influenza infection that seem to depend on the age of the host, the strain of the virus, environmental conditions, the degree of secondary bacterial infection, and many other variables. The clinical signs that typically appear, usually restricted to the upper respiratory tract, include the rapid onset of sneezing, nasal discharge, malaise, and pyrexia $^{1,3-7,9}$. Sometimes the signs are very mild and are not easily recognizable, whereas at other times the course of the disease results in a pneumonitis. In particular, the manifestation of pneumonitis seems to bear a close relation to the age of the host and/or the strain of the virus. Some strains are pneumotropic and cause a more severe form of influenza. Ferrets tend to show similar disease patterns to humans that are infected with the same type A strains $s^{1,7,9}$, and strains that produce pneumonitis in ferrets often produce pneumonitis in humans. Influenza in ferrets and humans infrequently culminates as an interstitial pneumonitis and is more commonly a rhinitis that progresses to tracheobronchitis. Influenza in ferrets and humans results in high morbidity and, in the young and immunocompromised, can be fatal. The influenza illness in both species is acute and usually lasts 3-5 days, so long as there are no complications. During this time, at the height of pyrexia, infected ferrets can transmit the disease to other ferrets and to humans. Aerosol droplets are a ready mode of transmission ${ }^{1,3,4,9}$.

Studies with ferrets have shown that the clinical signsespecially the pyrexia - that accompany influenza infection are the result of endogenous pyrogens released in response to the presence of the virus ${ }^{11-13}$. Investigations of the endogenous pyrogens in ferrets also proved important in clarifying the causes of human Reye's syndrome, which is caused by suppression of the fever that results from influenza infection. This syndrome can also develop with other viruses ${ }^{11}$.

\section{Pathogenesis}

The pathogenesis of the influenza virus in ferrets is very similar to that observed in humans ${ }^{3,14}$. When ferrets are experimentally inoculated intranasally, local replication of the virus in the upper airways occurs and, as discussed earlier, only rarely progresses to pneumonitis. Researchers have shown that whereas nasal turbinates are the primary site of viral replication, particularly virulent strains of influenza A are also capable of infecting the lower respiratory tract. The pathological changes seen in both ferrets and humans are most prominent in the upper airways; desquamation of nasal epithelium and infiltration of the submucosa of the nasal cavity with inflammatory cells typify the pathology ${ }^{3}$. Influenza virus selectively infects the ciliated respiratory epithelium in ferrets and humans. The influenza virus attaches to sialic acid on the surface of the respiratory epithelia via an $\alpha$-2,6-glycosidic linkage ${ }^{3}$. The importance of this linkage is that other influenza viruses, such as avian and equine influenza, link to respiratory epithelial cells by an $\alpha$-2,3-glycosidic linkage via sialic acid ${ }^{3}$.

Within $48 \mathrm{~h}$ after the infection of a ferret with a well-adapted strain, nearly complete destruction of the nasal respiratory epithelium occurs, leaving only the basement membrane ${ }^{15,16}$. Regeneration begins rapidly from the basement layer, and by the sixth or seventh day after infection, a transitional epithelium has already grown over the area ${ }^{15,16}$. Any of the variables mentioned earlier can influence the severity of the pathology. Research has shown that an influence on the severity of disease is the receptor specificity of the influenza A virus. As discussed earlier, the HA antigen is important for attaching and fusing to the cells, and thus minor changes to the amino acids at the receptor binding pocket may actually make the virus less efficient at attaching to the cell, resulting in a less virulent $\operatorname{strain}^{10}$. There is potential use for these receptor variants of the influenza virus for vaccine development, because some can elicit an immune response similar to that seen with the wild type.

\section{Immune Response}

Extensive study of the cellular immune system's response to the flu has shown it to be very similar in ferrets and humans,11,14,16-18. Studies using ferrets have shown that the cellular immune system is necessary for recovery from influenza infection ${ }^{14,18,19}$. The main cells that seem to be important to the cellular immune response are the cytotoxic lymphocytes, which attack infected cells expressing certain antigens $5,6,14,18,19$.

With respect to the immune system's humoral protection, one study showed that the presence of high serum antibody titers failed to protect the upper respiratory tract from viral challenge, even while it protected the lung and thus prevented lethal viral pneumonia $^{10}$. On the other hand, after neonatal ferrets suckled on an immune dam, milk-derived anti-influenza virus IgG (passive immunity) protected them against infection with influenza virus $^{19-24}$. IgA antibodies seem to have a role in protecting the upper respiratory tract in humans and other species ${ }^{20-23}$, and there has been an association of rapid recuperation in humans and other species with high titers of IgA in nasal washes.

\section{Strain Variations}

Some research studies have investigated the virulence of types A and $B$ influenza virus strains and variations of these strains. The ferret is a natural host of both the A and B type human influenza virus, but many of the studies done with ferrets have focused on the strains of the type A influenza virus ${ }^{25-29}$.

One aspect of influenza research for which ferrets have been used relates to deciphering age-related susceptibility to influenza infection, and many investigators have used the ferret model to 
study the relationship between age and humoral and cellular immunity. Understanding the body's defenses against the virus has proven extremely important for developing strategies to combat and resist the disease $\mathrm{e}^{27,30}$. In addition to these studies, the ferret model has played an important part in mapping out the pathogenesis of the typical influenza infection, transmission and reassortant variants of the virus, Reye's syndrome, drug treatment, and resistance of the influenza disease to many antiviral drugs $s^{30,31}$. Moreover, ferret models have served extensively in vaccine development, which in itself is a challenge because of the elusive behavior of the influenza type A virus, with its tendency to undergo frequent genotypic and phenotypic changes. In general, there has been so much use of the ferret in the research of the influenza virus that it would be impossible to summarize all aspects of the studies done with ferrets in the scope of this article.

\section{Anatomical and Physiological Characteristics}

There are physical characteristics that make the ferret an ideal model for deciphering the manifestations of influenza. First, the unusually long trachea of the ferret allows easy compartmentalization of the upper and lower respiratory tracts ${ }^{1,32}$, making it easier to study the effects of the virus on the different areas of the respiratory tract. Second, the ferret is a model large enough to allow easy monitoring of clinical parameters such as temperature, pulse, and respiratory rate. Third, they are the only small-animal model that develops a febrile response to naturally occurring influenza $\mathrm{A}^{6,32-34}$. Finally, the ferret is also a good model for studying influenza tissue tropism, because the swine, equine, and avian influenza virus, but not human influenza virus, replicate in the intestinal wall as well as the respiratory tract of ferrets ${ }^{1,33,34}$.

\section{Other Animal Models}

The ferret is a popular model for studies of the influenza virus, but it is not the only animal model available for these studies. Other models that have been used to study the influenza virus include the guinea pig, Syrian hamster, chinchilla, hedgehog, chicken, and rat ${ }^{1}$.

However, apart from the ferret, mice and certain nonhuman primate (NHP) species are most commonly the vehicle for studying the influenza virus. Because of its natural ability to contract the disease, the squirrel monkey often serves as the model for vaccine studies with reassortant type A influenza viruses. However, there are limitations to using this model, such as availability, the need for trained personnel, cost for caging and maintenance, additional health risks (e.g., TB), and the regulations associated with procuring and maintaining primates. Additionally, the lack of inbred NHPs makes using them difficult, because it increases the range of variables in the studies. On the other hand, the mouse, which is not a natural host of the virus, can still serve in studies of various aspects of the disease, and their small size and low cost allows researchers to conduct studies on a larger scale. That it is not a natural host to the virus limits its use, however. The mouse's small size also increases the difficulty of readily observing the clinical signs of the disease ${ }^{1}$.
As with any other laboratory animal model, there are also some disadvantages to use of the ferret. The initial cost of the animal is higher compared with some other models. Ferrets require more housing space than do mice, and equipment vendors do not generally sell cages for ferrets, so most facilities working with ferrets find that they must modify other cage types (e.g., rabbit, cat, or guinea pig cages) to accommodate them.

Another drawback to the use of ferrets is the lack of availability of inbred and specific pathogen-free ferrets. Because ferrets are not inbred, they do not all respond the same way to a particular strain of virus. Ferrets from standard vendors to be used for studies of influenza should first undergo testing to confirm that they do not have positive titers for the strain of influenza that is being studied. This in itself can be time consuming and add to the cost of the studies. Researchers often repeat these tests upon receiving the animals to ensure that they have not been infected during transport or reception. Because the animals come into contact with humans, a human who happens to be infected with influenza can potentially transfer the disease to the ferret, possibly complicating the studies. Some investigators suggest vaccinating staff against the influenza virus.

Finally, another disadvantage of using the ferret is the amount of labor required to care for them, especially as young animals. Because ferrets have a short small intestine, the intestinal transit time is correspondingly short. They also have a high metabolism rate and tend to eat frequently, and therefore they also defecate frequently. This situation leads to the need to spend additional time cleaning cages, so as to control the environment.

\section{Conclusions}

Studies of the influenza virus have made extensive use of the ferret, which has been instrumental in providing researchers with the ability to study various aspects of the disease as it relates to humans and in the development of viable treatments and vaccines to prevent the disease. As with any other animal model, the use of the ferret presents some drawbacks, but these are relatively minor considering the impact this model has had in the development of vaccines and treatments to improve the lives of both humans and animals.

Received 6/16/04; accepted 8/23/04.

\section{References}

1. Renegar, K.B. Influenza virus infections and immunity: a review of human and animal models. Lab. Anim. Sci. 42(3), 222-232 (1992).

2. Meltzer, M.I., Cox, N.J. \& Fukuda, K. The economic impact of pandemic influenza in the United States: priorities for intervention. Emerg. Infect. Dis. 5(5), 659-671 (1999).

3. Herlocher, M.L. et al. Ferrets as a transmission model for influenza: sequence changes in HA1 of type A (H3N2) virus. J. Infect. Dis. 184(5), 542-546 (2001).

4. Haff, R.F., Schriver, P.W. \& Stewart, R.C. Pathogenesis of influenza in ferrets: nasal manifestations of disease. Br. J. Exp. Pathol. 47(5), 435-444 (1966).

5. Lamb, R.A. \& Krug, R.M. in Fields Virology $4^{\text {th }}$ edn. Vol. 1 (eds. Knipe, D.M. \& Howley, P.M.) 1487-1531 (Lippincott Williams and Wilkins, New York, 2001).

6. Wright, P.F. \& Webster, R.G. in Fields Virology $4^{\text {th }}$ edn Vol. 1 (eds. 
Knipe, D.M. \& Howley, P.M.) 1533-1566 (Lippincott Williams and Wilkins, New York, 2001).

7. Baker, D.G. Natural Pathogens of Laboratory Animals: Their Effects on Research (ASM Press, Washington, DC, 2003).

8. Sakamoto, S., Kino, Y., Oka, T., Herlocher, M.L. \& Maassab, F. Gene analysis of reassortment influenza virus by RT-PCR followed by restriction enzyme digestion. J. Virol. Methods 56(2), 161-171 (1996)

9. Smith, H. \& Sweet, C. Lessons for human influenza from pathogenicity studies with ferrets. Rev. Infect. Dis. 10(1), 56-75 (1988).

10. Pearson C.R. \& Gorham J.R. in Biology and Diseases of the Ferret $2^{\text {nd }}$ edn (ed. Fox, J.G.) 487-497 (Lippincott Williams and Wilkins, New York, 1998).

11. Tinsley, C.M., Sweet, C., Coates, D.M. \& Smith, H. The local origin of fever in influenza: differential production of endogenous oxygen by nasal inflammatory cells of ferrets exhibiting different levels of fever. FEMS Microbiol. Lett. 42(2-3), 103-108 (1987).

12. Overton, H.A., Sweet, C., Coates, D.M. \& Smith, H. Molecular studies of the differential replication at pyrexial temperatures of two influenza viruses differing in virulence for ferrets. Virus Res. 5(2-3), 235-252 (1986)

13. Fenton, R.J., Clark, A. \& Potter, C.W. Immunity to influenza in ferrets. XIV: Comparative immunity following infection or immunization with live or inactivated vaccine. Br. J. Exp. Pathol. 62(3), 297-307 (1981).

14. Kauffman, C.A., Schiff, G.M. \& Phair, J.P. Influenza in ferrets and guinea pigs: effect on cell-mediated immunity. Infect. Immun. 19(2), 547-552 (1978).

15. Harper, S., Klimov, A., Uyeki, T. \& Fukuda, K. Influenza. Clin. Lab. Med. 22(4), 863-882 (2002).

16. Bartz, C.R. \& Montali, R.J. in Virus Infections of Carnivores (ed. Appel, M.J.) 445-448 (Elsevier, New York, 1987).

17. Barber, W.H. \& Small, P.A., Jr. Local and systemic immunity to influenza infections in ferrets. Infect. Immun. 21(1), 221-228 (1978).

18. McLaren, C. \& Butchko, G.M. Regional T- and B-cell responses in influenza-infected ferrets. Infect. Immun. 22(1), 189-194 (1978).

19. Collie, M.H., Rushton, D.I., Sweet, C. \& Smith, H. Studies of the influenza virus infection in newborn ferrets. J. Med. Microbiol. 13(4), 561-571 (1980).

20. Sweet, C., Jakeman, K.J., Rushton, D.I. \& Smith, H. Role of upper respiratory tract infection in the deaths occurring in neonatal ferrets infected with influenza virus. Microb. Pathog. 5(2), 121-125 (1988).

21. Sweet, C., Bird, R.A., Jakeman, K., Coates, D.M. \& Smith, H. Production of passive immunity in neonatal ferrets following maternal vaccinations with killed influenza A virus vaccines. Immunology 60(1) 83-89 (1987).

22. Jakeman, K.J., Rushton, D.I., Smith, H. \& Sweet, C. Exacerbation of bacterial toxicity to infant ferrets by influenza virus: possible role in sudden infant death syndrome. J. Infect. Dis. 163(1), 35-40 (1991).

23. Jakeman, K.J., Smith, H. \& Sweet, C. Mechanism of immunity to influenza: maternal and passive neonatal protection following immunization of adult ferrets with a live vaccinia-influenza virus haemagglutinin recombinant but not with recombinants containing other influenza virus proteins. J. Gen. Virol. 70(Pt. 6), 1523-1531 (1989).

24. Sweet, C., Toms, G.L. \& Smith, H. The pregnant ferret as a model for studying the congenital effects of influenza virus infection in utero: infection of fetal tissues in organ culture and in vivo. Br. J. Exp. Pathol. 58(2), 113-123 (1977).

25. Herlocher, M.L., Clavo, A.C. \& Maassab, H.F. Sequence comparisons of $A / A A / 6 / 60$ influenza viruses: mutations which may contribute to attenuation. Virus Res. 42(1-2), 11-25 (1996).

26. Kawaoka, Y, Bordwell, E. \& Webster, R.G. Intestinal replication of influenza A viruses in two mammalian species. Arch. Virol. 93(3-4), 303-308 (1987)

27. Watanabe, T., Watanabe, S., Kida, H. \& Kawaoka, Y. Influenza A virus with defective M2 ion channel activity as a live vaccine. Virology 299(2), 266-270 (2002).

28. Marsh, G.A., Watson, J.M., White, W.E. \& Tannock, G.A. An evaluation of the genetic stability and pathogenicity of the Russian coldadapted influenza A donor strains A/Leningrad/134/17/57 and A/Leninigrad/134/

$47 / 57 /$ in ferrets. J. Virol. Methods 107(1), 63-69 (2003).

29. Guo, Y. et al. Characterization of a new avian-like influenza A virus from horses in China. Virology 188(1), 245-255 (1992).

30. Herlocher, M.L. et al. Assessment of development of resistance to antivirals in the ferret model of influenza virus infection. J. Infect. Dis. 188(9), 1355-1361 (2003).
31. Burlington, D.B., Meiklejohn, G. \& Mostow, S.R. Toxicity of amantadine and rimantidine for the ciliated epithelium of tracheal rings. $J$. Infect. Dis. 144(1), 77 (1981).

32. Ball, R.S. Husbandry and management of the domestic ferret. Lab Anim. (NY) 31(5), 37-42 (2002).

33. Brown, S. in Ferrets, Rabbits and Rodents: Clinical Medicine and Surgery (eds. Hillyer, E.V. \& Quesenberry, K.E.) 3-13 (W.B. Saunders, Philadelphia, 1997)

34. Rosenthal, K.L. in Ferrets, Rabbits and Rodents: Clinical Medicine and Surgery (eds. Hillyer, E.V. \& Quesenberry, K.E.) 77-84 (W.B. Saunders, Philadelphia, 1997). 